\title{
Programming strategies and concepts for a CIM system
}

\author{
M.A. García, S. Alonso, K. Maraver, C. de Prada \\ Dpto. de Ingeniería de Sistemas y Automática \\ Facultad de Ciencias. Universidad de Valladolid \\ C/ Prado de la Magdalena, s/n.47005-Valladolid, Spain \\ Fax: +8342 31 61; e-mail:miguel@autom.uva.es
}

\begin{abstract}
A pilot plant for testing programming strategies for CIM systems is described. The main concepts are introduced and applied to the pilot plant, locating the problems, giving a particular solution and trying to generalise the basic lines of the programming process. Final results are discussed and their advantages are outlined.
\end{abstract}

\section{Keywords}

CIM, co-operative programming, distributed control, monitoring.

\section{INTRODUCTION}

Integrated solutions in manufacturing present two severe disadvantages in their practical implementation. On the one hand, the absence of industrial communication standards accepted by all the vendors and their commercial strategies lead to closed and proprietary solutions without the possibility of easily interconnecting different elements from different companies. On the other hand and as a consequence, generalised or rigorous approaches to manufacturing problems are not well-defined and are strongly dependent on specific components.

The pilot plant described in this paper was designed in order to explore the technology of CIM and for use in teaching students enrolled in courses on robotics and PLCs. The design of the pilot plant for testing the integration techniques took into account the pre-existence of some of the elements in the laboratory. Consequently, experience of working with an open and heterogeneous system was encountered from the first moment (defining openess as the possibility for elements from different vendors to interact, and the ability to change system components to ones providing increased performance, availability, etc., when the need arises (Rahkonen, 1995)). The function of the plant (see Figure 1) is a closed circuit for filling and unfilling bottles moving along a conveyor belt. The system consists of two computers (1), two Tecquipment robot arms (2) with their controllers (3), an Allen-Bradley PLC (15) and 
motion controller (16), a Bosch conveyor belt (4) with pallet (5), an SCS motor (7,8), servo (9) and tachometer (10), a Fagor encoder (6), an electro-valve in the hopper (18), presence detectors (14), and position switches $(11,12,13)$. There is a gravity channel (19) to retrieve the balls from the unloading zone and feed them to the hopper, and zones (17) with empty and full bottles. In spite of its lack of practical usefulness, this plant allows some of the fundamental concepts of communications and integration to be studied using a sequence of common activities in manufacturing.

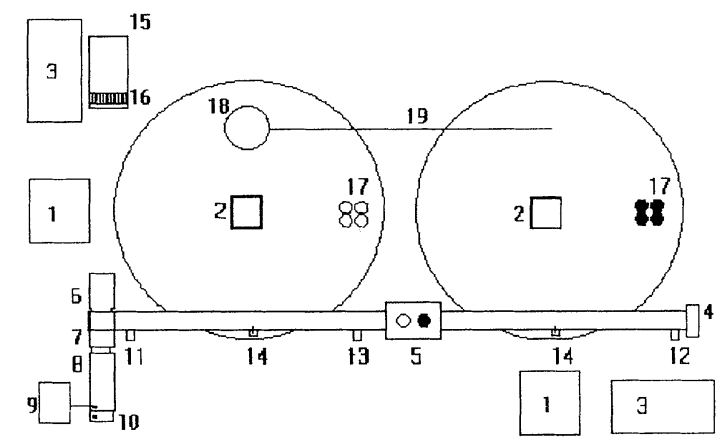

Figure 1 Pilot plant.

The paper is organised as follows: the two next sections describe how the concepts of 'fieldlevel' and 'plant-level' are applied to the pilot plant. Section 4 describes its communication system. The concepts of integration of the global problem and co-operative programming are focused on in sections 5 and 6, the latter introducing the 'function pairs' concept. Monitoring is briefly explained in section 7 , and section 8 presents some conclusions.

\section{FIELD LEVEL}

The first approach to the functionality of the pilot plant can be made at the so-called field level' (Popovic, 1990). For instance, if the pallet of the conveyor belt arrives at the end of its travel, an electrical switch will stop the movement. In the same way, the presence detectors for the bottles on the pallet operate directly as inputs to the robot controllers, and the PLC operates directly on the electro-valve. Field level communications, based on point to point communications, allow the basic operativity of the system and can be considered as a first step of integration. The CPUs in the system are directly connected to their sensors and actuators.

Obviously, this kind of communication has great limitations. There is no single point in the system where global information can be accessed, so decisions which are based on complex conditions of the entire plant cannot be taken. The field level allows only simple operations based on local information. 


\section{PLANT LEVEL}

At a higher level, the so-called 'plant level' can be considered. In outline, the plant level consists of a communications system between the CPUs. Although the best (and most common) way to achieve this communication is by using a bus, this is not strictly necessary. Open heterogeneous systems may contain CPUs which cannot easily share a common bus, and if there are no time constraints, almost the same functionality can be obtained with more than one bus or with point-to-point communications between pairs of CPUs.

The introduction of the plant level allows global information treatment, since the CPUs share their local information between them. Complex decisions can be taken based on information which is not necessarily local and enclosing actuators which are also not necessarily local, and advanced tools such us plant monitoring or fault detection can be offered to the user. As an example of high level fault detection, when a presence detector discovers the absence of a bottle on the pallet, the PLC can begin action to abort the sequence of movements of the robot trying to handle the missing bottle.

Although the information is centralised in some parts of the system, the behaviour of each CPU and its sensors and actuators follows the local control unit paradigm of distributed control, in such a way that simple decisions are taken locally.

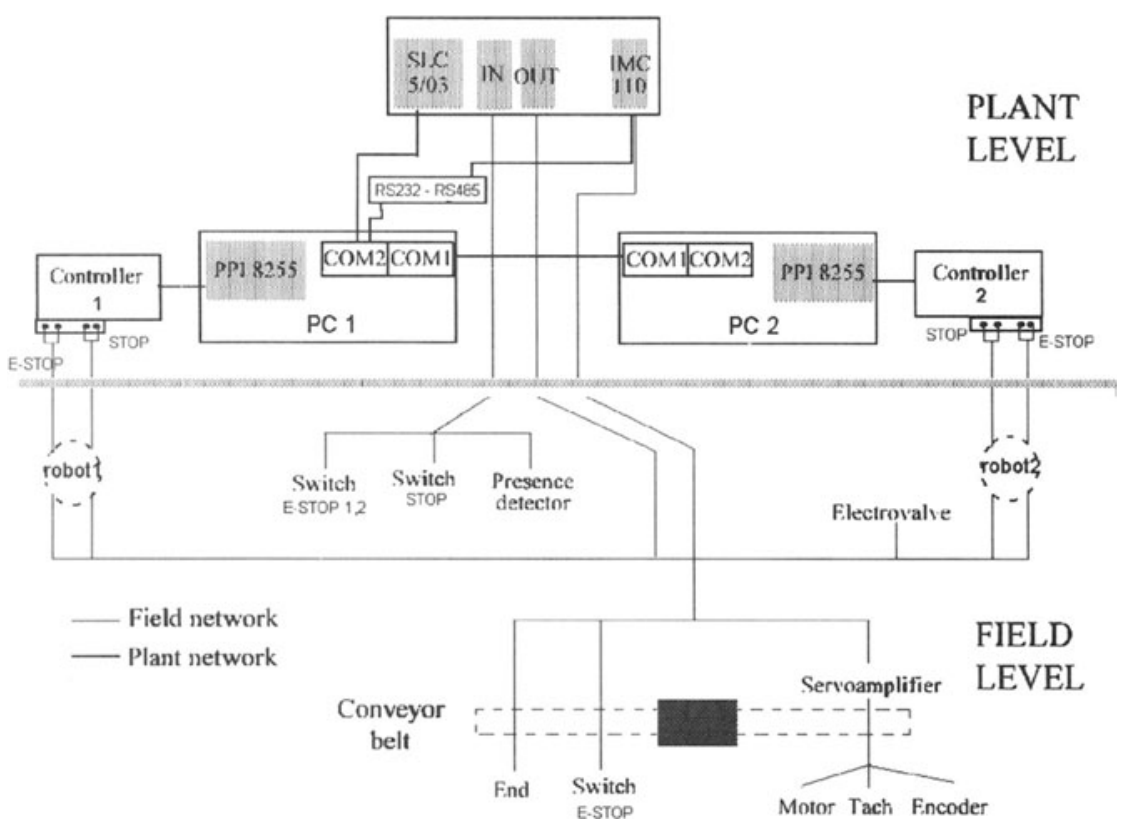

Figure 2 Levels structure. 


\section{COMMUNICATIONS SYSTEM}

The different kinds of CPUs in the pilot plant lead to a heterogeneous communication system. So, the communication between PC and robot controller is made through a peripheral interface card, while the communication PC-PC and PLC-PC is made through serial ports. Communication between PLC and motion controller is made through the backplane of the rack of the PLC, since the motion controller is a module plugged into the rack of the PLC. Such a set of communication subsystems makes it more difficult to share data between the CPUs, but by solving the communication problem for each pair of elements, information can flow throughout the system.

\section{INTEGRATION}

The most important idea in the approach to the system is to consider the problem of integration as a holistic one. In other words, a global view and analysis of the problem is needed before it can be divided into parts for each component in the system. In this way, the relations between the subproblems are well defined and are assigned places inside the total problem. In the pilot plant presented here, a GRAFCET (Arzen, 1995; Rahkonen, 1995) of the global system operation (see Appendix A) was designed. From the GRAFCET sequence, it is easier to assign the work to the different parts of the system, although it is not directly programmable with the elements mentioned in section 1 .

The motor of the GRAFCET of the system is the PLC. In fact, some kinds of PLCs (e.g. Telemecanique) can use GRAFCET as a programming language. In the pilot plant, the PLC is programmed in ladder logic, in such a way that a bit can be associated to all the transitions in the GRAFCET and each state has its own conditions, guaranteeing the sequenciality.

\section{CO-OPERATIVE PROGRAMMING}

Another important concept in an integrated system is co-operative programming. Although the ladder program in the PLC is driving the sequence, not all of the work can be made from this program. For instance, the PLC can close or open the electro-valve of the hopper, but it has no direct relationship with the user interfaces in the PCs, the motion of the conveyor belt or the operation of the robots. There are six microprocessors in the system with a variety of programming languages. Thus, the PCs execute $C$ language programs with assembler routines for communication with the controllers of the robots. The PLC is programmed with ladder logic, and the language of the motion controller is MML with AMP parameters (IMC110 (1) and (2)). All of them must work in co-operation, knowing the results of the others and offering their own information. The program for one of the elements cannot be made ignoring the programs for the rest, and all together must be considered part of the same programming project.

The way in which the PLC operates on the rest of the elements in the system and receives their data is by using a communication file in the file system of the program running in the PLC. This communication file is accessible via RS-232 from the PC linked to it. The PC has 
access to integer variables in the communication file in both directions, that is, reading and writing to it. The same applies to the motion controller, that uses an I/O file to store discrete data of the communication. Figure 3 shows how a STOP command is detected by the PLC program and how it operates on the rest of the programs working with it.

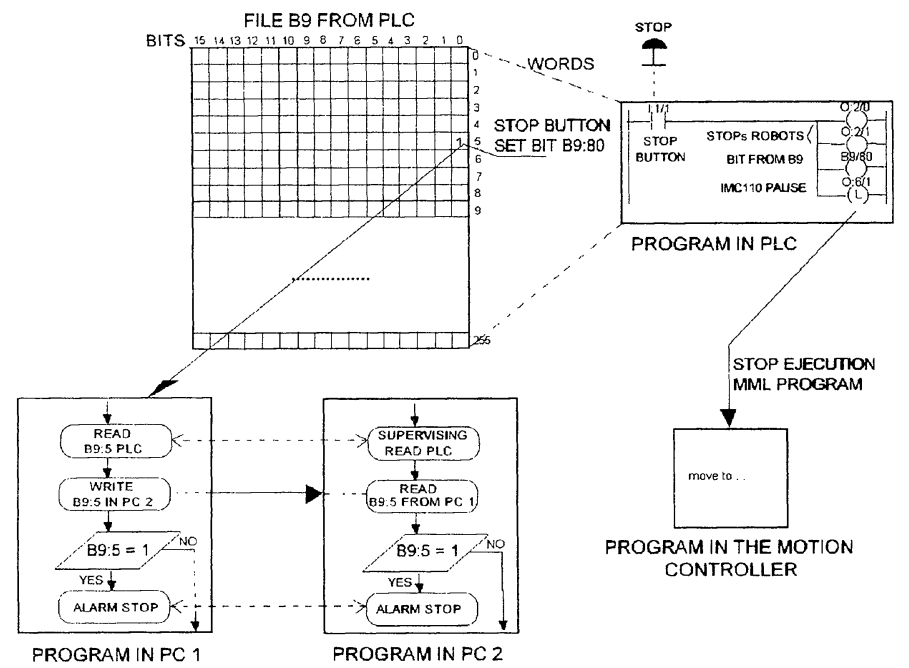

Figure 3 Co-operative programming.

The two PCs in the system carry out a set of duties. They supply the memory, storage and programming units for each of the robot controllers. Besides, they present interchangeable user interfaces concentrating the system information and allowing the user to choose between different options and to react to emergency situations. The programming tool for the robots is presented as part of the executable program in the $\mathrm{PC}$, and their movement sequences are integrated into the structure of stages directed by the PLC.

One 'stage' of the robot consists of one or more steps, which are the basic movement units of the robot. Stages and steps are stored dynamically in memory both during editing and execution time using linked structures in the $C$ language, and in independent files from one session to other. So, a collection of stages is available, and the order and instant of execution for each stage is determined by the PLC depending on information from its own program, the plant, the PCs or the motion controller. Each stage has a label, and the PLC puts these labels in order according to all the information arriving to it. The PLC communicates the chosen stage by writing its label to the PC linked to the robot. The PC then executes the corresponding sequence.

\subsection{Function pairs}

One of the main concepts in this implementation of co-operative programming is that of 'function pairs'. Each function in the pair is running simultaneously in each one of the PCs. Their purpose is to allow the monitoring of all the information in each PC, and to support the actions of the user wherever he/she is. One function in the pair carries out local actions in the $\mathrm{PC}$ in which it is executing, while the other function supervises those actions and allows their 
monitoring. Different pairs of functions are used for different treatments, active function in the pair being the function in one PC or in the other depending on the specific action. Usually the active function is in the PC physically attached to the PLC, because this PC manages more plant information.

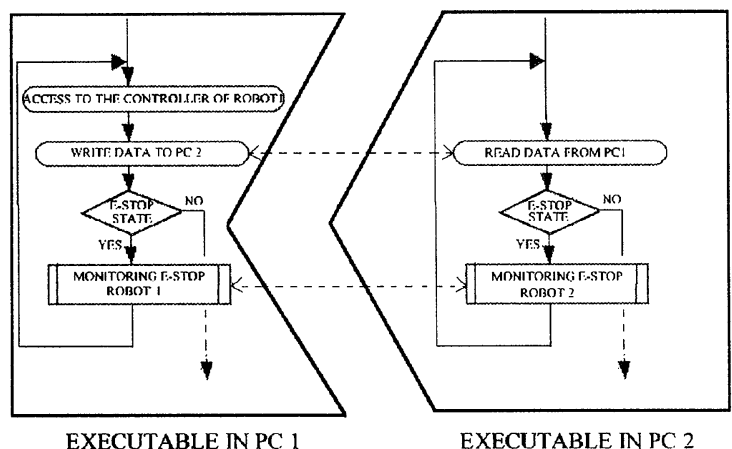

Figure 4 Function pairs.

\section{$7 \quad$ MONITORING}

The plant level communication makes the global information in the system accessible at different points. This fact allows the construction of a user interface on the PCs in the system (See Figure 5), taking advantage of the graphical possibilities of the $\mathrm{C}$ language. So, the PCs not only effect the treatment of the control information, but also use it to update the presentation in the monitor of the PC. Information about the current position of both robot arms, the pallet of the conveyor belt, the presence or absence of bottles on the pallet and the closing or opening of the electro-valve in the hopper are presented in a plan view of the system. This presentation is almost the same in both PCs. The only difference is the direct access to the robot, e.g. the programming tool and the recovery of E-STOPs (emergency STOPs). This kind of operation with the robots remains local for security reasons. In such a case, the interface presents a series of questions to the user, which must be answered for the recovery to take place.

\section{CONCLUSION}

A characteristic of CIM systems is that they are difficult to generalize. In spite of this, some strategies and considerations are useful in dealing with open manufacturing systems. The application of some of these strategies, such as the global approach, communication of the CPUs in the system and co-operative programming, has been explained. The benefits of such an approach, e.g. decisions based on complex conditions, fault detection and global monitoring have been developed on the pilot plant and briefly explained.

Although these kind of systems are almost impossible to configure automatically, using the strategies presented above, and other similar ones, a methodical approach to their implementation can be developed. 


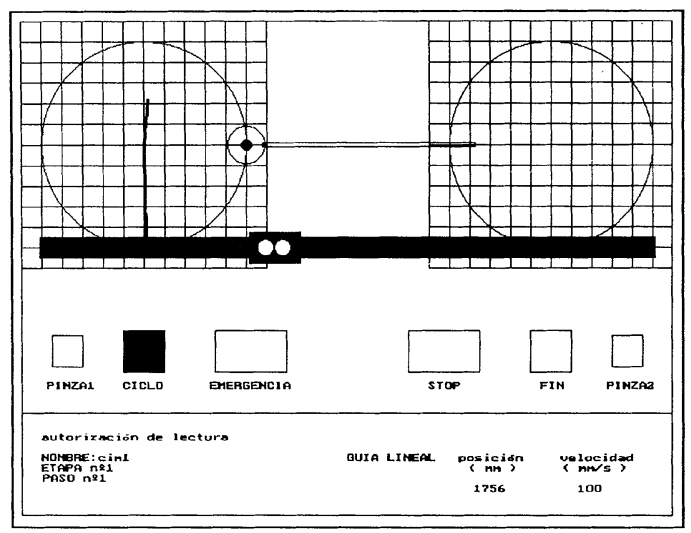

Figure 5 Monitoring.

\section{REFERENCES}

Arzen K.-E. (1995). GRAFCET for intelligent supervisory applications. Automatica, Vol. 30, $\mathrm{n}^{\circ} 10$, pp. 1513-1525.

Das S.K. (1992). A scheme for classifying integration types in CIM. International Journal CIM, vol. 5 , no. 1 .

Lecuivre J., Thomesse J.P. (1995). Definition of real-time services for heterogeneous profiles. Preprints DCCS'95, pp. 153-158.

Popovic D., Bhatkar, V.P. (1990). Distributed computer control for industrial automation. Marcel Dekker, Inc. USA.

Rahkonen T. (1995). Distributed industrial control systems. A critical review regarding openness. Control Engineering Practice, Vol 3, No 8, pp. 1155-1162.

Schoop R., Strelzoff A. (1995). Programming approaches for distributed control systems. Preprints DCCS'95, pp. 43-48.

IMC110 Motion controller system. Programming reference manual. Allen-Bradley.

IMC110 Motion controller system. AMP Reference manual. Allen-Bradley.

\section{BIOGRAPHY}

M.A. García is Physicist and Assistant teacher at the Dpto. de Ingeniería de Sistemas y Automática in the University of Valladolid. He is making his Ph. Degree in distributed control with real time constraints.

S. Alonso y K. Maraver are Technical Engineers in Telecommunications and have worked in the assemble of the plant and software components.

Professor César de Prada is director of Dpto. de Ingeniería de Sistemas y Automática in the University of Valladolid and has supervised the project. 


\section{APENDIX A: GRAFCET of the system}

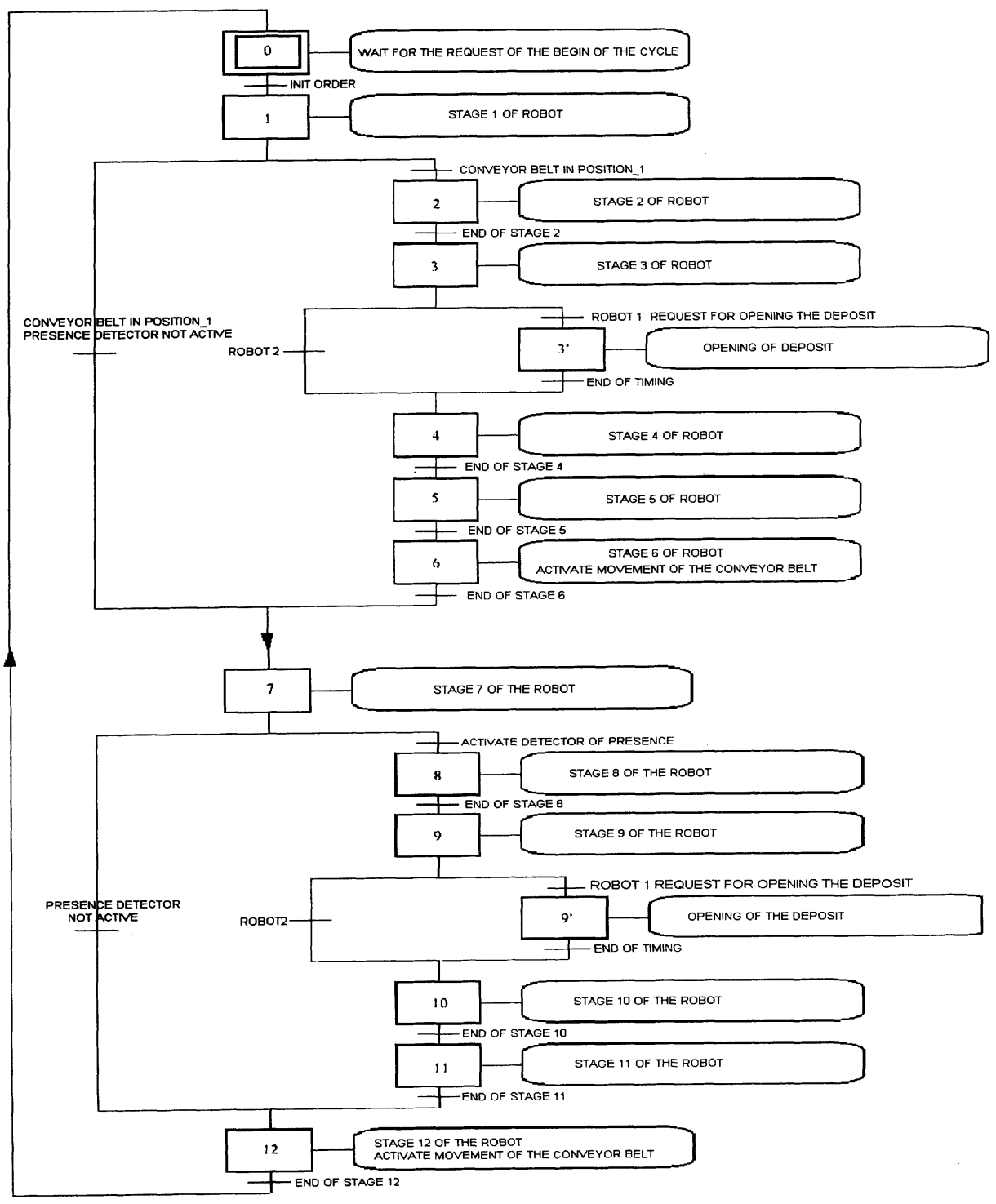

